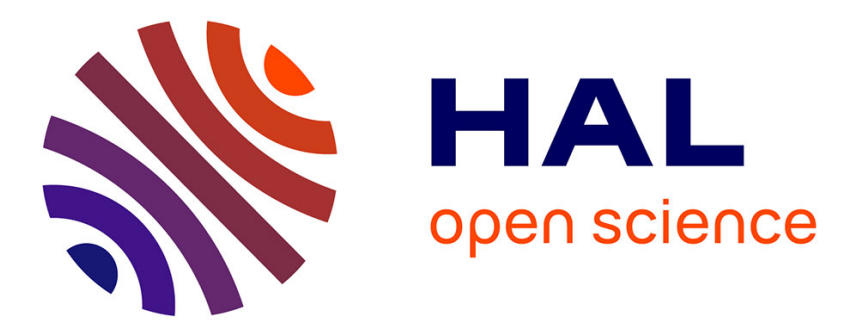

\title{
The influence of the chloride currents on action potential firing and volume regulation of excitable cells studied by a kinetic model
}

Nikolaus Berndt, Sabrina Hoffmann, Jan Benda, Hermann-Georg Holzhütter

\section{- To cite this version:}

Nikolaus Berndt, Sabrina Hoffmann, Jan Benda, Hermann-Georg Holzhütter. The influence of the chloride currents on action potential firing and volume regulation of excitable cells studied by a kinetic model. Journal of Theoretical Biology, 2011, 276 (1), pp.42. 10.1016/j.jtbi.2011.01.022 . hal-00682402

\section{HAL Id: hal-00682402 https://hal.science/hal-00682402}

Submitted on 26 Mar 2012

HAL is a multi-disciplinary open access archive for the deposit and dissemination of scientific research documents, whether they are published or not. The documents may come from teaching and research institutions in France or abroad, or from public or private research centers.
L'archive ouverte pluridisciplinaire HAL, est destinée au dépôt et à la diffusion de documents scientifiques de niveau recherche, publiés ou non, émanant des établissements d'enseignement et de recherche français ou étrangers, des laboratoires publics ou privés. 


\section{Author's Accepted Manuscript}

The influence of the chloride currents on action potential firing and volume regulation of excitable cells studied by a kinetic model

Nikolaus Berndt, Sabrina Hoffmann, Jan Benda, Hermann-Georg Holzhütter

PII: $\quad$ S0022-5193(11)00034-8

DOI: $\quad$ doi:10.1016/j.jtbi.2011.01.022

Reference: $\quad$ YJTBI6334

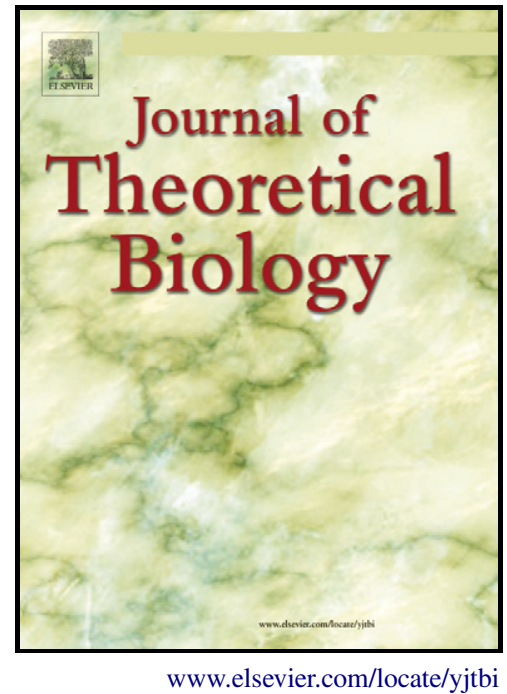

To appear in: $\quad$ Journal of Theoretical Biology

Received date: $\quad 30$ November 2010

Revised date: $\quad 13$ January 2011

Accepted date: 14 January 2011

Cite this article as: Nikolaus Berndt, Sabrina Hoffmann, Jan Benda and Hermann-Georg Holzhütter, The influence of the chloride currents on action potential firing and volume regulation of excitable cells studied by a kinetic model, Journal of Theoretical Biology, doi:10.1016/j.jtbi.2011.01.022

This is a PDF file of an unedited manuscript that has been accepted for publication. As a service to our customers we are providing this early version of the manuscript. The manuscript will undergo copyediting, typesetting, and review of the resulting galley proof before it is published in its final citable form. Please note that during the production process errors may be discovered which could affect the content, and all legal disclaimers that apply to the journal pertain. 


\section{The influence of the chloride currents on action potential firing and volume regulation of excitable cells studied by a kinetic model}

Nikolaus Berndt

Institute for Biochemistry,

Universitätsmedizin Berlin, Seestr. 73, 13347 Berlin, Germany

Sabrina Hoffmann

Institute for Biochemistry,

Universitätsmedizin Berlin, Seestr. 73, 13347 Berlin, Germany

Jan Benda

Department Biologie II,

Biozentrum der LMU, Grosshaderner Str. 2, 82152 Planegg-Martinsried, Germany

Hermann-Georg Holzhütter ${ }^{1}$

Institute for Biochemistry,

Universitätsmedizin Berlin, Seestr. 73, 13347 Berlin, Germany

${ }^{1}$ Corresponding author. Address: Institute for Biochemistry, Universitätsmedizin Berlin, Seestr, 73, 13347 Berlin, Germany, Tel.: +49 30450528166 


\begin{abstract}
In excitable cells, the generation of an action potential (AP) is associated with transient changes of the intra- and extracellular concentrations of small ions such as $\mathrm{Na}^{+}, \mathrm{K}^{+}$and $\mathrm{Cl}^{-}$. If these changes cannot be fully reversed between successive APs cumulative changes of trans-membrane ion gradients will occur, impinging on the cell volume and the duration, amplitude and frequency of APs. Previous computational studies focused on effects associated with excitation-induced changes of potassium and sodium. Here we present a model based study on the influence of chloride on the fidelity of AP firing and cellular volume regulation during excitation. Our simulations show that depending on the magnitude of the basal chloride permeability two complementary types of responsiveness and volume variability exist: (i) At high chloride permeability (typical for muscle cells), large excitatory stimuli are required to elicit APs; repetitive stimuli of equal strength result in almost identical spike train patterns (markovian behaviour), however, long excitation may lead to after discharges due to an outward directed current of intracellular chloride ions which accumulate during excitation; cell volume changes are large. (ii) At low chloride permeability (e.g., neurons), small excitatory stimuli are sufficient to elicit APs, repetitive stimuli of equal strength produce spike trains with progressively changing amplitude, frequency and duration (short-term memory effects or non-markovian behaviour); cell volume changes are small. We hypothesize that variation of the basal chloride permeability could be an important mechanism of neuronal cells to adapt their responsiveness to external stimuli during learning and memory processes.
\end{abstract}

Key words: chloride permeability; excitability; after discharge; stability of AP firing; short term memory 


\section{Introduction}

Action potentials (APs) represent elevations of the membrane potential from a typical resting value of about $-70 \mathrm{mV}$ to a peak value of about $+40 \mathrm{mV}$ within a few milliseconds, followed by an equally fast decline back to the resting value. During an AP, voltage-gated membrane channels for small ions as $\mathrm{Na}^{+}$and $\mathrm{K}^{+}$open and close in a timely well-coordinated fashion to enable transient extra currents of these ions along their concentration gradients: Sodium ions enter the cell and potassium ions flow into the extracellular space. Changes of the membrane potential feed back not only to voltage gated ion channels but also to the electro-diffusion of ions through passive channels, in particular chloride channels, which in most excitable cells possess a high resting conductivity. Shifts in the trans-membrane distribution of $\mathrm{Na}^{+}, \mathrm{K}^{+}$and $\mathrm{Cl}^{-}$occurring during an AP have to be reset by active ion transport. If this resetting has not been completed before a new AP is elicited, the perturbation of small-ion gradients across the membrane may successively progress during a series of APs (spike train).

Progressive changes of trans-membrane ion gradients during excitation may induce volume changes $(3,5,10)$. In the brain, swelling of neurons can be critical as it further reduces the small interstitial space and hence raises the external potassium concentration. This causes a shift of the membrane potential towards the firing threshold and thus increases excitability, which at the extreme can ignite seizures, spreading depression (SD) or hypoxic SD-like depolarization $(2,14)$.

For the regulation of the cell volume chloride is very prominent (13), as membrane currents of this negative and diffusible small ion may rapidly compensate additional anion currents. The basal chloride permeability is also an important determinant of the firing threshold. In many excitable cells as well as in the non-dendritic parts of the neuron, the cellular chloride concentration is mainly controlled by passive chloride currents implying that the reversal potential of chloride is very close to the cell's resting membrane potential. Thus, initial depolarization by excitatory cation currents causes an counteracting inward directed chloride current. As a consequence, in order to reach a depolarization that is sufficient to open voltage gated sodium channels, increasing chloride permeabilities entail higher excitatory currents. An important exception from this general behaviour is the postsynaptic membrane of GABAergic neurons. Here, active transport of chloride is indispensable for the corresponding membrane region to either hyperpolarize (inhibitory synapse) or depolarize (excitatory synapse) upon neurotransmitter-gated opening of chloride channels $(6,15,22,23)$. 
In this work we present a detailed computational study on the impact of chloride currents on the volume regulation and firing pattern of excitable cells. The mathematical model used to simulate temporal changes in the intra- and extracellular concentrations of $\mathrm{Na}^{+}, \mathrm{K}^{+}$and $\mathrm{Cl}^{-}$couples changes of ion concentrations to trans-membrane ion currents mediated by active ion pumps, non-gated and voltage-gated ion channels and membrane permeabilities. The simulations correctly recapitulate observed volume changes of active neuronal cells and the hyper-excitability of muscle cells underlying the neuromuscular disease myotonia congenita. The simulations also predict a number of novel and hitherto experimentally unstudied effects that a variable passive chloride permeability may have on the frequency, shape and duration of spike trains. Based on our theoretical findings we hypothesize that variations of the chloride permeability may represent a hitherto underestimated mechanism to tailor neuron responsivness to specific profiles of excitatory stimuli.

\section{Description of the mathematical model}

The model describes the dynamics of the three ion species $\mathrm{Na}^{+}, \mathrm{K}^{+}$and $\mathrm{Cl}^{-}$in a model cell characterized by its spherical geometry and the types, membrane densities and kinetic properties of ion channels and ion pumps (in the following referred to as 'cell').

The model takes into account three types of trans-membrane ion transports: (i) passive ion transport mediated by non-gated ion channels, (ii) passive ion transport through voltage-gated (excitatory) ion channels and (iii) active transport of sodium and potassium by the Na-K-ATPase (Fig. 1).

The time-dependent variation of ion concentrations is described by the following kinetic equations:

$$
\begin{aligned}
\frac{\mathrm{d}[X]_{\text {cell }}}{\mathrm{d} t} & =-\frac{I_{X}}{z_{X} F \Omega_{\text {cell }}}-\frac{[X]_{\text {cell }}}{\Omega_{\text {cell }}} \frac{\mathrm{d} \Omega_{\text {cell }}}{\mathrm{d} t}, \\
\frac{\mathrm{d}[X]_{\text {out }}}{\mathrm{d} t} & =\frac{I_{X}}{z_{X} F \Omega_{\text {out }}}-\frac{[X]_{\text {out }}}{\Omega_{\text {out }}} \frac{\mathrm{d} \Omega_{\text {out }}}{\mathrm{d} t} .
\end{aligned}
$$

Here $F$ is Faraday's constant, $\Omega_{\mathrm{c}}$ and $\Omega_{\text {out }}$ denote the cell volume and the volume of the extracellular space, $z_{X}$ denotes the valence and $I_{X}$ denotes the net trans-membrane current of ion species $X \in\left\{\mathrm{Na}^{+}, \mathrm{K}^{+}, \mathrm{Cl}^{-}\right\}$obtained as sum of the current $I_{x}^{\mathrm{p}}$ associated with passive ion transport (i.e., transport driven by electro-diffusion) and the current $I_{X}^{\mathrm{a}}$ maintained by active ion transport driven by energy-dependent pumps: 


$$
I_{X}=I_{X}^{\mathrm{a}}+I_{X}^{\mathrm{p}} .
$$

The passive ion current $I_{X}^{\mathrm{p}}$ is described by a Goldman-Hodgkin-Katz equation

$$
I_{X}^{\mathrm{p}}=-A \Pi_{X} U F z_{X}^{2} \frac{[X]_{\mathrm{cell}} \exp \left(z_{X} U\right)-[X]_{\mathrm{out}}}{1-\exp \left(z_{X} U\right)},
$$

where $A$ is the membrane area, $U=\frac{F V}{R T}$ and $\Pi_{X}$ being the total membrane permeability for ion species $X$ brought about by all passive ion transport processes. We decompose the total membrane permeability additively into three contributions:

$$
\Pi_{X}=P_{X}^{0}+P_{X}^{\mathrm{g}}+\Delta P_{X} .
$$

$P_{X}^{0}$ denotes the basal permeability for ion species $X$ related to all kinds of passive membrane transport except transport through voltage-gated channels. Note that $P_{X}^{0}$ also includes membrane permeability due to ion-coupled substrate transporters as, for example, uptake of neurotransmitters. $P_{X}^{\mathrm{g}}$ is the time- and membrane potential-dependent permeability of gated ion channels for ion species $X$. Our model contains gated channels for $\mathrm{Na}^{+}$and $\mathrm{K}^{+}$which we model in the same way as in the standard model of Hodgkin and Huxley (12):

$$
\begin{aligned}
P_{\mathrm{K}}^{\mathrm{g}} & =m^{4} P_{\mathrm{K}}^{\mathrm{g} 0}, \\
P_{\mathrm{Na}}^{\mathrm{g}} & =n^{3} h P_{\mathrm{Na}}^{\mathrm{g} 0} .
\end{aligned}
$$

The gating variables $g \in\{h, m, n\}$ obey ordinary differential equations

$$
\frac{\mathrm{d} g}{\mathrm{~d} t}=C_{g}\left(\alpha_{g}(1-g)+\beta_{g}\right) .
$$

$C_{g}$ is a constant and $\alpha_{g}$ and $\beta_{g}$ represent membrane potential dependent functions which have to be specified for each ion species and the type of sodium and potassium channel considered (see appendix). The third term in equation (5), $\Delta P_{X}$, accounts for changes in the membrane permeability for species $X$ elicited by both, ligand-gated ion channels and fluctuations of passive non-gated ion transports (e.g., ion gradient-driven substrate transporters).

The active transport of $\mathrm{Na}^{+}$and $\mathrm{K}^{+}$is accomplished by the Na-KATPase. The 'standard' rate equation for the Na-K-ATPase is 


$$
\begin{aligned}
I_{\mathrm{K}-\text { ATPase }}^{\mathrm{a}} & =2 F \frac{k_{\mathrm{NaK}}}{\left(1+\frac{\mathrm{Na}_{c}}{\left[\mathrm{Na}^{+}\right]_{\mathrm{cell}}}\right)^{n}}, \\
I_{\mathrm{Na}-\text { ATPase }}^{\mathrm{a}} & =-3 F \frac{k_{\mathrm{NaK}}}{\left(1+\frac{\mathrm{Na}_{c}}{\left[\mathrm{Na}^{+}\right]_{\mathrm{cell}}}\right)^{n}},
\end{aligned}
$$

where the pump is considered as saturated with ATP (the Michaelis constant for ATP is in the range of $300-500 \mu \mathrm{M}(21)$ and thus far below the normal cellular ATP level). The regulatory influence of $\left[\mathrm{K}^{+}\right]_{\text {out }}$ is neglected and the dependence from $\left[\mathrm{Na}^{+}\right]_{\text {cell }}$ is taken into account as a Hill-type sigmoidal function with $n>1$. The parameter $k_{\mathrm{NaK}}$ denotes the maximal rate of the pump and $\mathrm{Na}_{\mathrm{c}}$ the half saturation constant for sodium.

To relate the membrane potential $V$ to the trans-membrane currents, the membrane is regarded as a capacitor with a total capacitance $C$ given by

$$
C=c_{\mathrm{m}} A=\frac{Q}{V}
$$

where $Q$ is the total charge on the capacitor and $V$ is the membrane potential generated by this charge, $c_{\mathrm{m}}$ is the specific membrane capacitance and $A$ denotes the cell's surface. We assume that membrane convolution and deconvolution prevents changes of the surface area $A$ at moderate changes of the cell volume. Differentiation of relation (11) with respect to time yields

$$
\frac{\mathrm{d} V}{\mathrm{~d} t}=\frac{1}{C} \frac{\mathrm{d} Q}{\mathrm{~d} t}-\frac{Q}{C^{2}} \frac{\mathrm{d} C}{\mathrm{~d} t}
$$

Neglecting time-dependent variations of the membrane capacitance, i.e., putting $\frac{\mathrm{d} C}{\mathrm{~d} t}=0$, we get

$$
\frac{\mathrm{d} V}{\mathrm{~d} t}=\frac{1}{C} \sum_{X} \frac{\mathrm{d} Q_{X}}{\mathrm{~d} t}=\frac{1}{C} \sum_{X} I_{X} .
$$

Finally we have to set up an equation relating the cellular volume changes to the internal and external ion concentrations. Osmotic forces arising from a difference in inner and outer osmolarity, $\mathrm{O}_{\text {in }}$ and $\mathrm{O}_{\text {out }}$, are counterbalanced by a fast trans-membrane exchange of water resulting in changes of the cell volume: 


$$
\begin{aligned}
\frac{\mathrm{d} \Omega_{\text {cell }}}{\mathrm{d} t} & =P_{\mathrm{w}}\left(\mathrm{O}_{\text {in }}-\mathrm{O}_{\text {out }}\right), \\
\mathrm{O}_{\text {in }} & =\sum_{X}\left(\left|z_{X}\right|[X]_{\text {cell }}\right)+[\mathrm{B}], \\
\mathrm{O}_{\text {out }} & =\sum_{X}\left(\left|z_{X}\right|[X]_{\text {out }}\right), \\
\frac{\mathrm{d} \Omega_{\text {out }}}{\mathrm{d} t} & =-\frac{\mathrm{d} \Omega_{\text {cell }}}{\mathrm{d} t} .
\end{aligned}
$$

$P_{\mathrm{w}}$ is the water permeability and [B] denotes the contribution of cellular non-diffusible but osmotic active particles (cellular biomass) to the total intracellular osmolarity.

All parameters, a brief description of their meaning, the numerical values used in the simulations and the references to the sources are depicted in the appendix. The parameter values were calibrated such that typical values for the intra- and extracellular ion concentrations and the membrane potential established at resting conditions $\left(\Delta P_{X}=0\right.$ in equation (5)) are obtained.

For the integration of the differential equations the MATLAB software 6.5.1.199709 (R13) Service Pack 1 was used.

\section{Results}

\section{The role of chloride in the regulation of cell volume}

\section{Volume regulation of non-excitable cells}

To illustrate the general role of chloride fluxes in changes of the cellular volume, we first applied our model to a non-excitable cell by putting the permeability constants for the gated potassium and sodium channels to zero, $P_{\mathrm{K}}^{\mathrm{g} 0}=P_{\mathrm{Na}}^{\mathrm{g} 0}=0$. In this case, our model comprises the same membrane processes as the model that Armstrong (4) proposed to simulate the impact of the Na-K-ATPase and chloride on the osmotic stabilization of the cell. It has to be noted, however, that our simulations include temporal changes of the membrane potential according to equation (13) whereas in the Armstrong model the non-adequate equilibrium assumption $\mathrm{d} V / \mathrm{d} t=0$ was made.

Fig. 2 illustrates the importance of the chloride current on changes of the cellular volume after inhibition of the Na-K-ATPase. If chloride channels are blocked, only positively charged ions may enter the cell. This causes a depolarization of the membrane, which then prevents further positive ions 
from entering the cell. Due to the low specific capacitance of the membrane, only very few ions are needed to depolarize the membrane. Thus change of internal osmolarity and the accompanied cell volume change is negligibly small. In contrast, if chloride is allowed to enter the cell, a massive influx of positive and negative ions compensating each other's charges occurs. Although the flux of net charge across the membrane is very small, the 'osmotic current' is fairly high giving rise to cell volume changes. The swelling rate increases with increasing chloride permeability. A decrease of the basal chloride permeability delays cell swelling, a fact that has inspired experimental studies in which inhibitors of chloride transport were applied to alleviate osmotic changes under conditions of ischemia-induced energy depletion of neurons (17).

\section{Volume regulation of excitable cells}

We next performed simulations of volume changes in an excitable cell at varying magnitudes of the basal chloride permeability. Excitation was achieved by increasing the permeability of the sodium channel over the time period indicated. During excitation the membrane potential is on the average less negative than at resting conditions. Hence, negative chloride ions enter the cell and the cell volume increases (see Fig. 3 A). The simulations shown in Fig. $3 \mathrm{~B}$ and $3 \mathrm{C}$ demonstrate that volume changes during excitation scale with the basal chloride permeability (determining the magnitude of the inward directed passive chloride current) and the duration of excitation. The lower the basal chloride permeability the smaller the rate of cell swelling. This is analogous to the observation in non-excitable cells (Fig. 2). Our simulations suggest that the passive chloride permeability of excitable cells has to be kept sufficiently low to prevent sizeable volume changes during AP firing. At a basal chloride permeability of $40 \cdot 10^{-9} \frac{\mathrm{m}}{\mathrm{s}}$ a volume increase of more than 5 percent is achieved in less than one minute (Fig. 3 B). Considering that the extracellular volume in neuronal tissues amounts to only 20 percent (19), a 5 percent increase in the volume of neurons can lead to 25 percent compression of the extracellular volume which is accompanied by an equally large increase of extracellular ion concentrations, in particular potassium. This may result in seizure-like after-discharges $(2,3)$. 


\section{The influence of chloride currents on the characteristics of the firing pattern}

In order to study the influence of the basal chloride permeability on the frequency and duration of AP firing we performed simulations where we varied the passive chloride permeability between 0 and the 2 -fold of the reference value. With increasing passive chloride permeability the frequency of APs changed dramatically (Fig. 4). At passive chloride permeabilities lower than the reference value, the AP frequency increased by more than 50 percent, while at 1.5 fold increased basal chloride permeability just a single AP was elicited. At two fold increase no action potentials were elicited at all.

This remarkable dependence of AP firing on the basal chloride permeability can be understood as follows: Initial depolarization causes an inward directed chloride current that counteracts the excitatory sodium current. Thus, in order to reach a depolarization that is sufficient to open voltage gated sodium channels, increasing chloride permeabilities entail higher excitatory sodium currents. As the sodium gradient decreases progressively during excitation, the excitatory sodium current will ultimately drop below the value required to elicit further AP firing. Thus, the higher the passive chloride permeability, the higher the required initial excitatory current, and therefore, the longer the time period within which the sodium gradient remains sufficiently steep. This situation is illustrated in (Fig. 5), where we chose the permeability of the excitatory sodium current for 20 seconds $(=$ duration of the excitation period) either 1 percent or 66 percent above the minimal value required to elicit excitation at a passive chloride permeability of $10 \cdot 10^{-9} \frac{\mathrm{m}}{\mathrm{s}}$. It is clearly seen that the duration of AP firing increased with higher excitation permeability. Nevertheless, even at an excitation permeability that is 1.66 fold higher than the minimal threshold value firing stopped before the end of the excitation period. In other words, for an excitable cell to fire for a prescribed duration, the excitation permeability (the strength of the necessary excitatory sodium permeability) is determined by the absolute value of the basal chloride permeability (Fig. 6). Intriguingly, for basal chloride permeabilities below a critical value of $40 \cdot 10^{-8} \frac{\mathrm{m}}{\mathrm{s}}$, the necessary excitatory sodium permeability depends upon the duration of AP firing, whereas for basal chloride permeabilities above this threshold the necessary excitatory sodium permeability becomes independent from the duration of AP firing. Taking together, these simulations show that a high basal chloride permeability stabilizes AP firing.

An intriguing consequence of the above simulation findings is that a de- 
crease of the basal chloride permeability may confer a short-term memory to excitable cells in that their firing response to a series of repetitive excitatory stimuli of equal strength is progressively changing (Fig. 7 A). Depending on the number of preceding stimuli and the time gap between them, the duration of AP firing can be substantially shortened. Such firing behaviour is called non-markovian, because the response of the excitable cell depends on its excitation history. In contrast, high basal chloride permeabilities require high excitatory sodium currents (see above) and thus enable a markovian response, i.e., a practically constant 1:1 transmission of repetitive stimuli into spike trains (Fig. 7 B)

Our simulations also provided evidence that a high chloride permeability can not only stabilize AP firing, but can also pose a major threat to a cell, not only by enhancing cell swelling, but also by causing a chloride induced after discharge (see Fig. 8 B2). During AP firing intracellular chloride accumulates, since more chloride enters the cell during membrane depolarization than leaves the cell during hyperpolarization. The total amount of intracellular chloride accumulation depends on the absolute value of the passive chloride permeability. When the excitation stops, chloride leaks out of the cell and decreases the excitation threshold. This chloride-induced afterdischarge is fundamentally different from the well known potassium-induced after-discharge in neurons which is caused by high extracellular potassium concentrations (see Fig. 8 A2). Whereas the latter results from the collective contribution of simultaneous active neurons and depends strongly upon the size of the extracellular space and the capacity of neighboring glia cells to remove extracellular potassium (8), the chloride-dependent discharge predicted by the simulations in Fig. 8 B2 is an intrinsic feature of a single excitable cell.

\section{Discussion}

Every action potential is accompanied by a transient perturbation of transmembrane ion distributions. Incomplete nullification of this perturbation between successive action potentials may give rise to cell swelling and distort the shape and frequency of action potentials or the duration of AP firing. Such alterations would interfere with the capability of an excitable cell to respomd in a precise and reproducible manner to incoming electric or humoral stimuli. To address this issue we have set up a mathematical model that allows us to simulate the impact of various trans-membrane ion transport processes on the fidelity of long-term action potential firing at con- 
stant excitation. We focused on the functional role of chloride in osmotic regulation and AP firing.

The mathematical model used in the work considers the small ion concentrations as uniformly distributed across the cell's cytosol. However, larger variations in the intracellular spatial distribution of small ions as, for example, calcium have been reported (9). In order to check whether the kinetic phenomena studied in this work are sensitive against the presence of an intra-cellular concentration gradients of small ions, we extended our model by introducing an additional small sub-membrane space (a thin shell, whose size was chosen according to the protrusion of the Na-K-ATPase into the cell) adjacent to the inner cell membrane (see supplement). Ion exchange between this small sub-membrane compartment and the interior bulk phase took place via diffusion. Simulations of this extended model showed regular fluctuations of ion concentrations in the sub-membrane space that were in phase with temporal changes of the membrane potential. These fluctuations diffusionally propagated into the bulk phase where they were largely damped out due to the slowness of the diffusion process and the much bigger volume of the bulk space. However, over time scales much longer than a single action potential, the concentration changes within the bulk phase obtained with the 2-compartment model were only marginally different from those obtained with the more simple 1-compartment model. Hence, taking explicitly into account the non-uniform spatial distribution of small ions resulted in only small quantitative but not qualitative alterations of all kinetic phenomena reported in this work.

As in non-excitable cells (Fig. 2) changes in cell volume, induced by changes of the activity of trans-membrane ion gradients, are caused by passive chloride currents (see Fig. 3). Our simulations suggest that under normal physiological conditions excitation-induced cell swelling is in the range of a few percent. For neuronal cells, such changes are confirmed by experimental studies (7). Significant changes of the cellular volume can be observed under conditions of a sustained increase in intensity of ongoing neuronal activity, reflected by increased gamma-frequency EEG power (20). Excitation-induced massive increase of intracellular chloride has even been implicated in the neurotoxicity of amino acids, such as glutamate, N-methylo-aspartate, and kainate (18).

Another intriguing consequence of excitation-induced passive chloride currents is the modulation of the frequency and long-term maintenance of spike trains. Our simulations suggest that at chloride permeabilities below a critical value and for low excitation currents, the duration of AP firing is controlled by the magnitude of the excitatory sodium current (see Fig. 6), 
a situation more likely to be found in neurons. In contrast, if chloride permeability is more than about twice as high as potassium permeability or even higher, a situation met by muscle (11), higher excitatory sodium currents are required, which, once established, are only marginally attenuated through the decrease of the sodium gradient during excitation. Intriguingly, in muscle cells, where chloride contributes about $2 / 3$ of the attenuation of the initial membrane depolarization (exerted by all passive ion currents), impaired chloride permeability leads to myotonia (16). In this neuromuscular disease, the chloride permeability of the skeletal muscle sarcolemma is drastically diminished due to a mutation of the chloride channel ClC-1 which normally possesses a high chloride permeability and thus stabilizes the resting potential. Reduced permeability of the mutated channel results in a hyper-excitable membrane and altered firing patterns $(1,13)$. The lower basal chloride permeability of neurons compared to muscle cells may be attributed to the limited space available. As a higher basal chloride permeability leads to a higher rate of cells swelling (Fig. 3), the low chloride permeability may be a necessity to avoid the compression of the interstitial space and the associated danger of spreading depression (2).

Our computational finding that variations in the passive chloride permeability of excitable cells may strongly influence the duration, frequency and reproducibility of spike trains may have also relevance for the design of neuronal networks. For example neurons with a very low basal chloride permeability have a comparable small excitation threshold, but their firing duration will depend on the input strength. As a result repetitive stimuli of identical strength and length will result in spike trains with successively declining amplitudes and shortened duration of firing (Fig. 7). Hence, the response of such neurons depends on their excitation history, a short-term memory behaviour usually termed non-markovian. On the other hand, neurons with high chloride permeability do not show such memory effects, but instead respond to repetitive stimuli with an almost unchanging spike train pattern.

The main goal of our study was to investigate the impact that variations in the interplay of basal permeabilities, ion concentrations and active ion transport may have on the stability and characteristics of spike trains and the regulation of the cell volume during excitation. Our intention was to unravel basic mechanisms rather than providing the most realistic simulation of real cells. Accordingly, we used a conceptual model that lumps the large variety of reported channels and transporters for $\mathrm{Na}^{+}, \mathrm{K}^{+}$and $\mathrm{Cl}^{-}$into a small set of overall processes. Our simulations revealed that temporary trends of the trans-membrane distributions of $\mathrm{Na}^{+}, \mathrm{K}^{+}$and $\mathrm{Cl}^{-}$ 
during excitation and the accompanying changes of the cell volume and the characteristics of the spike trains are intrinsic to the basic architecture of the membranous AP generating system and thus have to occur irrespective of the simplicity and specific parameterization of the chosen mathematical model. Altering the kinetic properties of channels or pumps, or including other types of ions and channels can only impact on the extent of these characteristics.

\section{References}

[1] Adrian, R. H. and Bryant, S. H. (1974), J Physiol 240(2), 505

[2] Andrew, R. D. (1991) , J Neurol Sci 101(1), 7

[3] Andrew, R. D. and MacVicar, B. A. (1994), Neuroscience 62(2), 371

[4] Armstrong, C. M. (2003), Proc Natl Acad Sci US A 100(10), 6257

[5] Ballanyi, K., Grafe, P., Serve, G., and Schlue, W. R. (1990), Glia 3(3), 151

[6] Coull, J. A. M., Boudreau, D., Bachand, K., Prescott, S. A., Nault, F., Sk, A., Koninck, P. D., and Koninck, Y. D. (2003) , Nature 424(6951), 938

[7] Darqui, A., Poline, J. B., Poupon, C., Saint-Jalmes, H., and Bihan, D. L. (2001), Proc Natl Acad Sci U S A 98(16), 9391

[8] Fröhlich, F., Bazhenov, M., Iragui-Madoz, V., and Sejnowski, T. J. (2008) , Neuroscientist 14(5), 422

[9] Harary, H. H. and Brown, J. E. (1984), Science 224(4646), 292

[10] Hill, D. K. (1950) , J Physiol 111(3-4), 304

[11] Hodgkin, A. L. and Horowicz, P. (1959) , J Physiol 148, 127

[12] Hodgkin, A. L. and Huxley, A. F. (1952), J Physiol 117(4), 500

[13] Jentsch, T. J. (1996) , Curr Opin Neurobiol 6(3), 303

[14] Kager, H., Wadman, W. J., and Somjen, G. G. (2007) , J Comput Neurosci 22(2), 105 
[15] Kahle, K. T., Staley, K. J., Nahed, B. V., Gamba, G., Hebert, S. C., Lifton, R. P., and Mount, D. B. (2008) , Nat Clin Pract Neurol 4(9), 490

[16] Koch, M. C., Steinmeyer, K., Lorenz, C., Ricker, K., Wolf, F., Otto, M., Zoll, B., Lehmann-Horn, F., Grzeschik, K. H., and Jentsch, T. J. (1992) , Science 257(5071), 797

[17] Müller, M. (2000) , Neuroscience 97(1), 33

[18] Rothman, S. M. (1985) , J Neurosci 5(6), 1483

[19] Sykov, E. (2005), J Neural Transm 112(1), 137

[20] T. Olsson, M. Broberg, K. P. A. W. L. M. F. B. M. N. and Willoughby, J. (2006) , Neuroscience Volume 140, Issue 2, 2006, Pages 505-515 Volume 140, Pages 505

[21] Therien, A. G. and Blostein, R. (2000) , Am J Physiol Cell Physiol 279(3), C541

[22] Woo, N.-S., Lu, J., England, R., McClellan, R., Dufour, S., Mount, D. B., Deutch, A. Y., Lovinger, D. M., and Delpire, E. (2002) , Hippocampus 12(2), 258

[23] Zhu, L., Polley, N., Mathews, G. C., and Delpire, E. (2008) , Epilepsy Res 79(2-3), 201 


\section{Figure Legends}

\section{Figure 1.}

Schematic representation of the processes included into the mathematical model

The diameters of passive ion channels symbolize relative ion permeabilities of $\mathrm{Cl}^{-}, \mathrm{Na}^{+}$and $\mathrm{K}^{+}$(40:0.8:20). Excitation is achieved by increasing the permeability of the Na channel (= excitatory permeability) mimicking the opening of voltage- or ligand gated $\mathrm{Na}$ channels. The action potentials are accomplished by the voltage-gated channels for sodium $\left(\mathrm{Na}_{\text {gated }}\right)$ and potassium $\left(\mathrm{K}_{\text {gated }}\right)$. The trans-membrane gradients of $\mathrm{Na}^{+}$and $\mathrm{K}^{+}$are maintained by the ATP-driven Na-K-ATPase giving rise to a trans-membrane potential that is negative at the cytosolic side. Osmotic equilibrium between the extra- and intracellular space is established by the exchange of water through water channels. The net charge of non-diffusible organic cellular molecules is taken into account by the variable 'biomass'.

\section{Figure 2 .}

Simulated time course of ion concentrations and volume changes of a nonexcitable cell at varying chloride permeabilities

The permeabilitiy constants of voltage-gated channels were set to zero (=non-excitable cell). As chloride is only passively exchanged the chloride current equals zero at resting conditions, i.e., blocking of the passive chloride permeability (at the time point indicated by the first vertical dashed line) did not change the chloride concentration. Shut-down of the Na-K-ATPase (at the time indicated by the second vertical dashed line) caused an initial jump in the membrane potential, due to the cessation of the outward directed net transport of positive charges by the electrogenic pump. This was followed by further membrane depolarization and a massive change in the intracellular concentrations of $\mathrm{Na}^{+}$and $\mathrm{K}^{+}$, but the cell volume remained constant. Removing the blockade of the chloride channel (at the time point indicated by the third vertical dashed line) led to an influx of $\mathrm{Cl}^{-}$and continuous cell swelling until theoretically the volume is infinite. These changes were less pronounced if the initial chloride permeability were only partially restored (transparent curves), i.e., the swelling rate depends on the absolute value of the chloride permeability. 


\section{Figure 3.}

Simulated time course of ion concentrations, volume changes and firing pattern of an excitatory cell at varying chloride permeability

Between time $t=0.5 \mathrm{sec}$. and $t=5.5 \mathrm{sec}$. ( $=5 \mathrm{sec}$. excitation period) the sodium permeability was set from the resting value $8 \cdot 10^{-9} \frac{\mathrm{m}}{\mathrm{s}}$ to 10.32 . $10^{-9} \frac{\mathrm{m}}{\mathrm{s}}$. The ratio between the extra- and intracellular volume was set to 10 resulting in an only moderate change of the external ion concentrations. After $2.5 \mathrm{sec}$. of excitation (at $t=3 \mathrm{sec}$.) the passive chloride permeability was put to zero. Gray bars indicate the time spans in which the excitatory sodium permeability was not equal to zero.

A: The excitatory sodium current initiated AP firing with a rate of $\sim 45 \mathrm{~Hz}$. Within 2.5 seconds of excitation, the cellular volume increased by about 0.4 percent. Putting the passive chloride permeability to zero stoped cell swelling, but the firing frequency was elevated to $\sim 80 \mathrm{~Hz}$. AP firing was accompanied by a continuous change of internal and external ion concentrations.

B: Dependence of volume changes on the duration of the excitation period for different constant chloride permebilities.

C: Volume increase after a firing period of 20 and 40 sec. as function of the passive chloride permeability.

All numerical values of model parameters used in the simulations are listed in the supplement.

\section{Figure 4.}

Influence of the passive chloride permeability on the firing pattern

The excitatory permeability of the sodium channel was set to a constant value $2.5 \cdot 10^{-9} \frac{\mathrm{m}}{\mathrm{s}}$ for $0.2 \mathrm{sec}$. (=length of the excitation period indicated by the grey background). The basal chloride permeability was varied in 5 steps between 0 and $80 \cdot 10^{-9} \frac{\mathrm{m}}{\mathrm{s}}$ (values given in the upper left corners of the 5 pannels). Increase of the chloride permeability successively diminished the frequency of action potentials which finally ceased completely at $80 \cdot 10^{-9} \frac{\mathrm{m}}{\mathrm{s}}$.

\section{Figure 5.}

Dependence of the firing duration on the strength of the excitatory input

The excitatory-Na-permeability was chosen for $20 \mathrm{sec}$. (= length of the excitation period marked by the grey background) either as 1.01-fold (A) or 1.66 -fold of the excitatory threshold value. The basal chloride permeability was $10 \cdot 10^{-9} \frac{\mathrm{m}}{\mathrm{s}}$. The duration of AP firing increased with input strength, 
but even an excitation strength 66 percent above the threshold value was insufficient to trigger action potentials for the whole excitation period of 20 sec. The ratio between the extra- and intracellular volume was put to 100 resulting in negligible small changes of external ion concentrations.

\section{Figure 6.}

The basal chloride permeability determines the minimal excitatory sodium permeability required to assure AP firing over a prescribed duration

The solid lines indicate the relation between the basal chloride permeability (horizontal axis) and the minimal excitatory sodium permeability (vertical axis) needed to trigger repetitive APs for at least 1 second (open circles), 5 seconds (open squares), 10 seconds (stars), 20 seconds (crosses) and 40 seconds (diamonds). There is a monotonic relation between basal chloride permeability and the required minimal permeability of the excitatory $\mathrm{Na}$ current. Intriguingly, the minimal permeability of the excitatory $\mathrm{Na}$ channel required for stable AP firing becomes independent of the prescribed duration of firing, if the basal chloride permeability exceeds a critical value of $40 \cdot 10^{-8} \frac{\mathrm{m}}{\mathrm{s}}$. In contrast, below this critical threshold the duration of AP firing depends on the input strength.

\section{Figure 7.}

Firing response of an excitable cell to repetitive excitatory stimuli of constant strength

AP firing was elicited by repetitive excitatory stimuli of constant strength sufficient to elicit AP firing for at least 6 seconds starting from resting conditions, each stimulus having a duration of 1.5 seconds and being separated from the preceding stimulus by a non-stimulatory period of 1.5 seconds.

A: Firing response of an excitable cell with a low chloride permeability of $10 \cdot 10^{-9} \frac{\mathrm{m}}{\mathrm{s}}$. The firing frequency decreased. The stimulus was eventually insufficient to trigger AP firing over the whole stimulation period.

B: Firing response of an excitable cell with a high basal chloride permeability of $60 \cdot 10^{-9} \frac{\mathrm{m}}{\mathrm{s}}$. The firing frequency increased due to internal chloride and external potassium accumulation.

\section{Figure 8.}

Different types of simulated after discharges

(A) Potassium-induced after discharge provoked by changing the ratio of intracellular to extracellular space from 1:10 (A1) to 1:2 (A2). The duration 
of constant excitation (grey) was 20 seconds. AP frequency increased continuously due to external potassium accumulation. At the end of the stimulation enough external potassium has accumulated in panel A2 to cause after discharge.

(B) Chloride-induced after discharge provoked by increasing the basal chloride permeability from $40 \cdot 10^{-9} \frac{\mathrm{m}}{\mathrm{s}}$ (B1) to $70 \cdot 10^{-9} \frac{\mathrm{m}}{\mathrm{s}}$ (B2) while keeping all other model parameters constant. The ratio of intracellular to extracellular space was set to 1:1000 to exclude any influence of accumulating extracellular potassium. The duration of constant excitation (grey) was 60 seconds. AP frequency increased due to intracellular chloride accumulation. At the end of the stimulation enough internal chloride had accumulated in panel B2 to cause after discharge.

The panels below the spike trains (second and fourth row) show the time course of the corresponding internal $\mathrm{Cl}^{-}$and external $\mathrm{K}^{+}$concentrations.

(C) Panel C1 shows the spike train from A2 at the time of stimulus end. Due to the sudden decrease of the excitatory sodium permeability there was an abrupt decrease in AP firing frequency, but the external potassium concentration was high enough to cause after discharges. Panel C2 shows the same situation for the chloride induced after discharge from B2. 


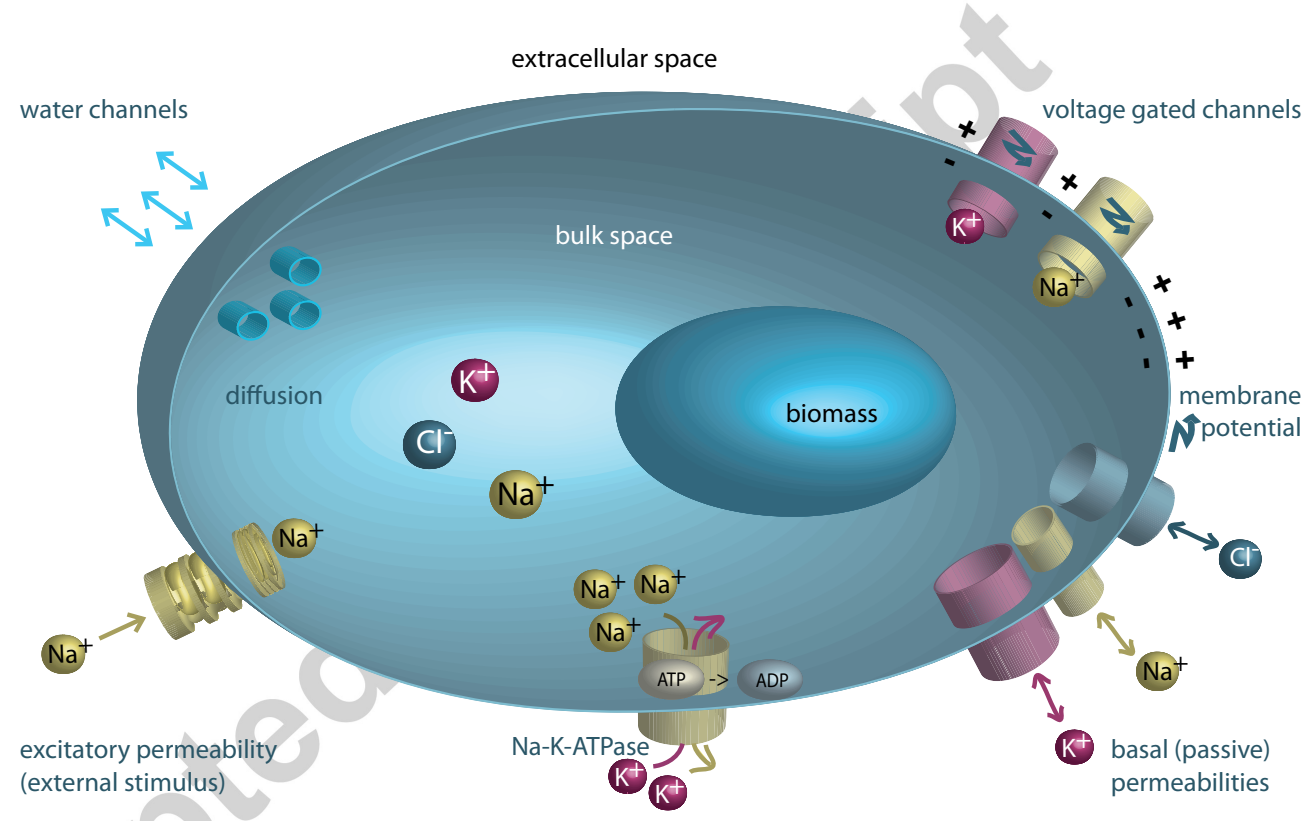

Figure 1: 


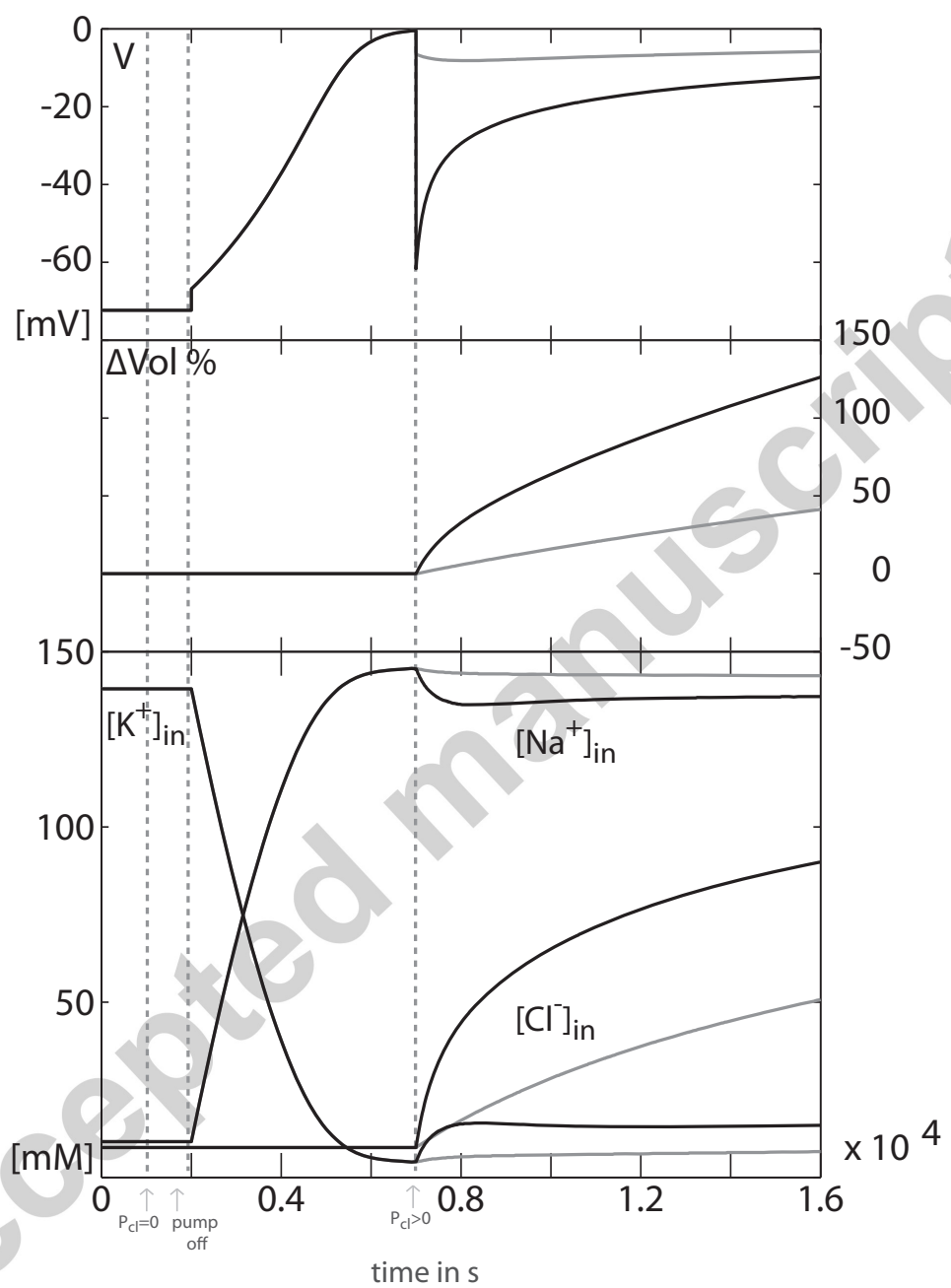

Figure 2: 

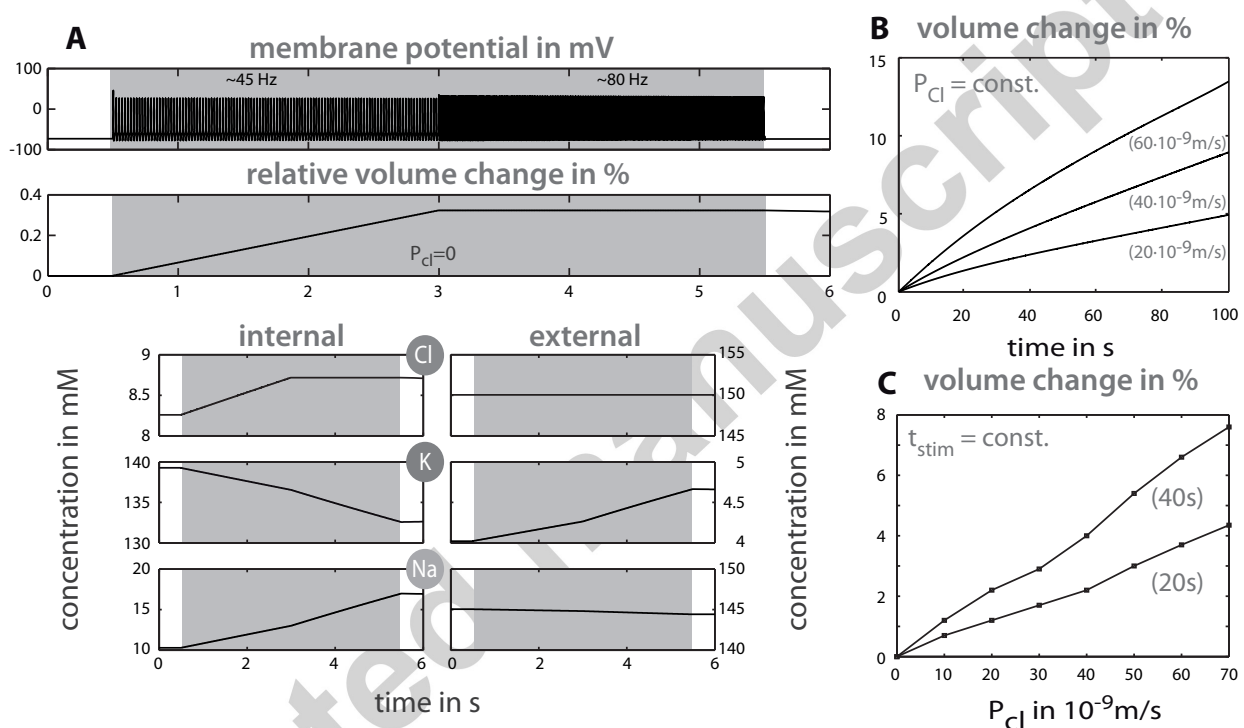

Figure 3: 
[mV] chloride permeability in $10^{-9} \mathrm{~m} / \mathrm{s}$

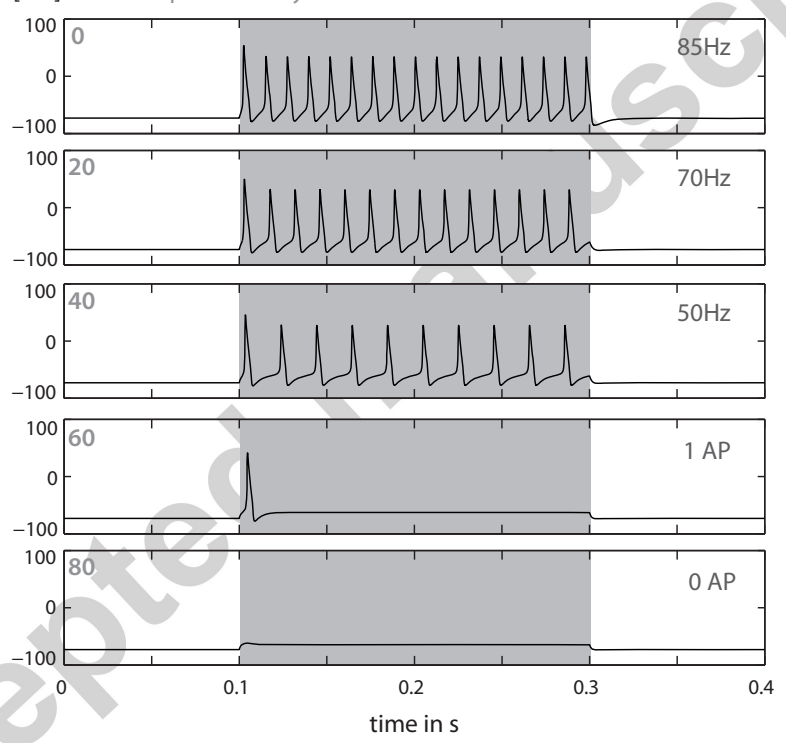

Figure 4: 


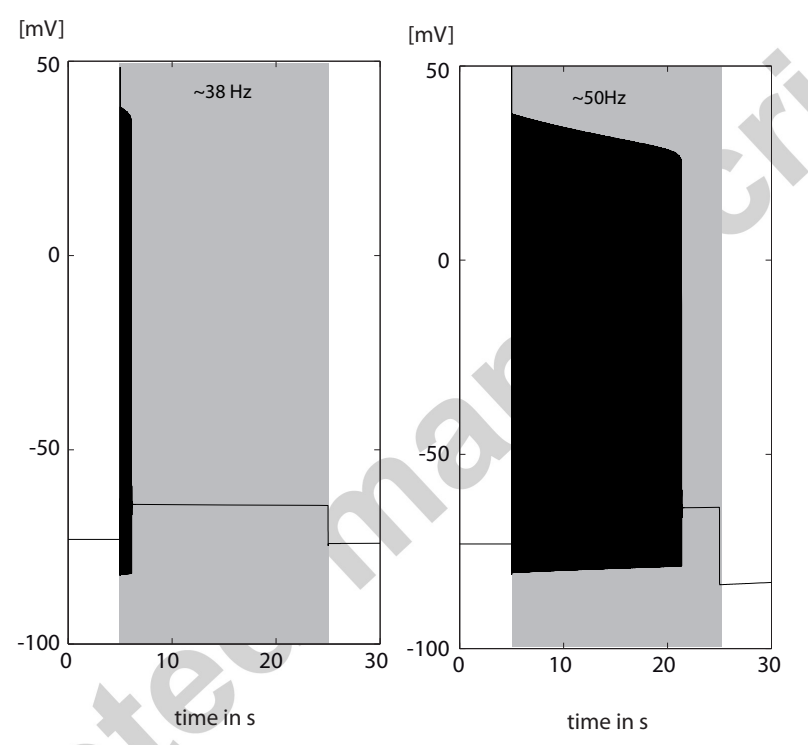

Figure 5: 


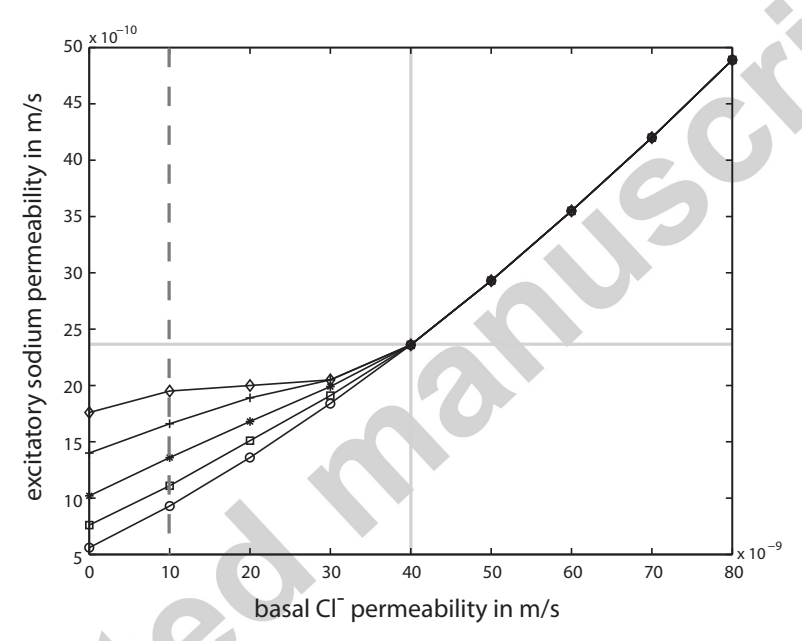

Figure 6: 


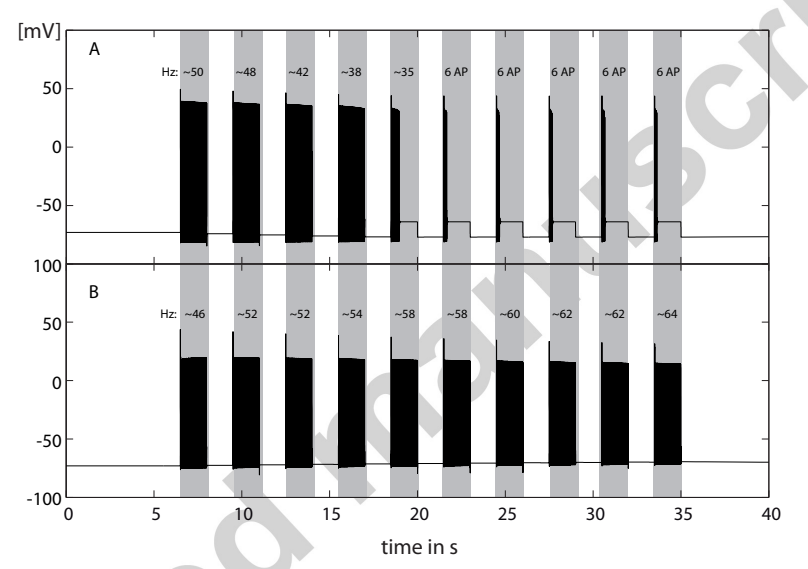

Figure 7: 

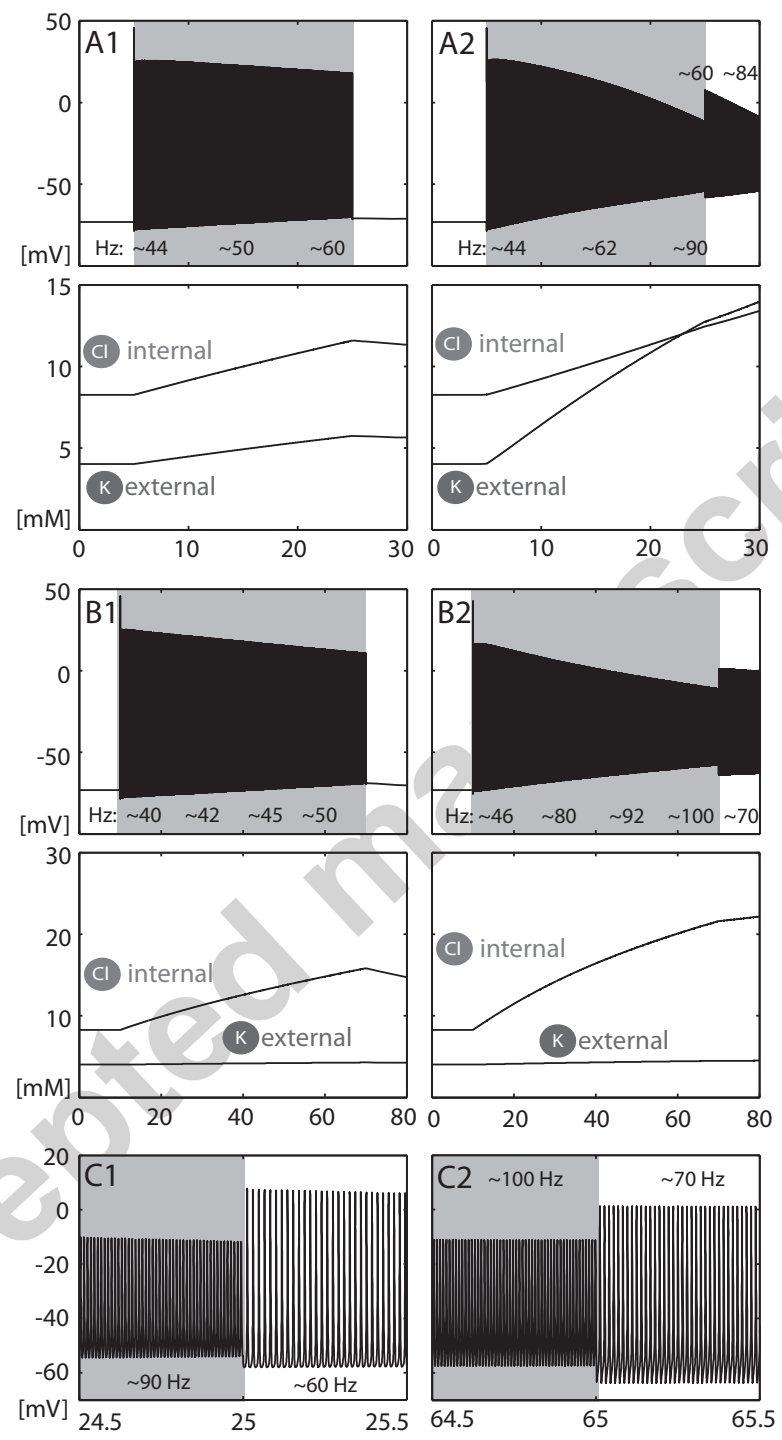

Figure 8: 